\title{
Cognitive remediation, brain function and central coherence: an anorexia nervosa pilot study
}

\author{
Leon Fonville ${ }^{1 \dagger}$, Vincent Giampietro ${ }^{2 \dagger}$, Helen Davies ${ }^{1}$, Naima Lounes ${ }^{1}$, Andrew Simmons ${ }^{2,3}$, Steven Williams $s^{2,3}$ \\ and Kate Tchanturia ${ }^{1,4^{*}}$
}

\begin{abstract}
Objectives: Cognitive remediation therapy (CRT) for anorexia nervosa (AN) is an intervention designed to improve the thinking processes of AN patients as well as their general cognitive functioning. While previous behavioural studies have shown promising results, no studies to date have assessed the neuronal effects of CRT in AN.

Methods: In this study, nine patients and fourteen healthy controls (HC) performed a variant of the embedded figures test (EFT) during functional magnetic resonance imaging at two separate occasions to assess central coherence. Patients received 10 sessions of CRT in between scans, and controls did not receive any training.

Results: While both groups showed improvement on the task over time, there was no indication of greater improvement in performance after CRT. Neuroimaging data did reveal that on complex embedded figures (CEF), those with AN showed a stronger decrease in task-related activation during the follow-up scan in the fusiform gyrus and middle occipital gyrus as well as greater task-related deactivation in the medial frontal gyrus extending into the precuneus.

Conclusions: This greater neural efficiency in AN after CRT could be indicative of successfully adopting a more global strategy on the EFT and suggests that CRT affects central coherence on a neural level.
\end{abstract}

Keywords: Anorexia, fMRI, Cognitive remediation therapy, Attention to detail, Central coherence

\section{Introduction}

Anorexia nervosa (AN) is a psychiatric disorder that affects almost $1 \%$ of young women [1] and is associated with high mortality rates [2] and poor prognosis [3]. Treatment is often problematic [4] with high drop-out rates and limited acceptance and adherence to treatment. This is often attributed to the inflexible and obsessional cognitive style found in patients [5].

Previous research has suggested a neurocognitive profile illustrating inefficiencies in cognitive flexibility, attention, central coherence, social cognition and long-term and visuospatial memory [6-9], but these neuropsychological processes are generally not addressed in treatment. Cognitive remediation therapy (CRT) aims to raise awareness of thinking styles and to improve strategies through series of exercises designed to develop a more flexible and global

\footnotetext{
*Correspondence: kate.tchanturia@kcl.ac.uk

${ }^{\dagger}$ Equal contributors

'Department of Psychological Medicine, King's College London, Institute of Psychiatry, De Crespigny Park, London SE5 8AF, UK

${ }^{4}$ Illia State University, Cholokashvili 5, 0162 Tbilisi, Georgia

Full list of author information is available at the end of the article
}

thinking style [6,9]. CRT has been implemented in individual and group settings, both for outpatients and inpatients, with promising results (for review, see [10]). This is illustrated by reports of positive qualitative feedback [11], low drop-out rates [12] and medium to large effect size improvements in cognitive task performance [13].

To date, there have been no studies that have assessed the effects of CRT in AN using neuroimaging. Studies of CRT in schizophrenia have reported improvements in domains such as working memory, attention, social cognition and reasoning, alongside changes in brain activation [14-17] as well as preservation of brain structure and volume $[17,18]$.

This study aimed to assess the effects of CRT in AN on central coherence using functional magnetic resonance imaging (fMRI). Central coherence, as measured by a variant of the embedded figures test (EFT), was chosen as a previous cross-sectional investigation of this paradigm found better performance in healthy controls $(\mathrm{HC})$ with less neural effort, indicative of utilizing a more global approach on this paradigm [19]. Many of the exercises in CRT focus on 
teaching patients to adopt a more global approach [20,21], but their effects have not been evaluated using fMRI.

We hypothesized that after CRT, patients with AN would employ a more global approach on the EFT, defined as an improvement in accuracy and reaction time and a decrease in neural activation in regions associated with object recognition and visuospatial searching.

\section{Methods}

\section{Participants}

Nine patients with a current diagnosis of AN fulfilling DSM-IV criteria (age $=22$, BMI (before/after CRT) $=16 / 15.7$, duration of illness $=3$ years), who previously participated in neuroimaging studies at the Institute of Psychiatry, were recruited to undergo 10 sessions of CRT over a period of 2 months at the Eating Disorders Service at the Maudsley Hospital or the Bethlem Royal Hospital, both part of the South London and Maudsley NHS Foundation Trust (SLaM). Successful completion of all 10 sessions was followed by a follow-up brain scan. Details of CRT administration are described in [21]. Five patients reported taking antidepressant medication throughout the duration of the study. Fourteen HC (age $=24, \mathrm{BMI}=22.1$ ), who had previously participated in the same studies as the AN group, were rescanned without any form of training between the initial (T1) and follow-up scan (T2). Nine HC were selected for further comparison with the AN sample after optimal matching in terms of age and IQ using MatchIt [22,23] in $\mathrm{R}$ [24]. The participants were predominantly right-handed, although five left-handed participants took part in the study (three AN and two HC). Consent was obtained according to the Declaration of Helsinki and was approved by the National Research Ethics Committee London Bentham (11-LO-0952).

\section{Paradigms}

On the Embedded Figures Tests (described in detail in [19]), the participants were required to indicate which of two figures contained a simple geometrical shape by moving a joystick to the left or to the right. The level of difficulty varied, and the figures were either simple (SEF) or complex (CEF) in composition (see [25] for further details). There were 18 unique trials for both the SEF and the CEF, each lasting for a total of $10 \mathrm{~s}$, and were presented in alternating blocks of three. The order of the blocks did not change. A white fixation cross on a black background, utilized as a low-level baseline, was presented for $30 \mathrm{~s}$ at the start, middle and end of the experimental run. The total runtime of the experiment was 450 s. The paradigm did not differ between the two time points. Performance data (reaction time and accuracy) were analysed using SPSS 20 (IBM [26]). As the data were non-normal, the KruskalWallis test incorporating the Scheirer-Ray-Hare extension [27] was used as a non-parametric two-way ANOVA (as described in [28]), with group (HC and AN) and time (T1 and T2) as factors. Task complexity (SEF and CEF) was not added as a factor due to a clear ceiling effect during SEF across the whole sample at both time points.

\section{MRI acquisition and processing}

Magnetic resonance imaging was performed using a $1.5 \mathrm{~T}$ GE Signa HDx TwinSpeed MRI scanner (GE-Medical Systems, WI, USA) at the Centre for Neuroimaging Sciences, Institute of Psychiatry, King's College London. Details of the MRI acquisition and of the image analysis are described in detail in earlier reports from our group [19]. Group $\times$ time interaction analyses were performed to assess if there was a difference in improvement over time between the two groups on either the SEF or the CEF. A false discovery rate (FDR) of less than one false positive per brain activation map was utilized to correct for multiple comparisons.

\section{Results}

\section{EFT performance and imaging results}

Clinical measures in AN did not differ from $\mathrm{T} 1$ to $\mathrm{T} 2$, and $\mathrm{HC}$ scores did not reach clinical cut-off. There was no statistically significant change in the body mass index (BMI) in either group. All clinical measures are summarised in the first section of Table 1. On SEF, trends for a main

Table 1 Overview of clinical measures and performance on the EFT for patients with AN and HC

\begin{tabular}{|c|c|c|c|c|}
\hline & \multicolumn{2}{|l|}{$\mathrm{AN}(n=9)$} & \multicolumn{2}{|c|}{$\mathrm{HC}(n=9)$} \\
\hline & T1 & $\mathrm{T} 2$ & T1 & $\mathrm{T} 2$ \\
\hline \multicolumn{5}{|c|}{ Clinical measures } \\
\hline BMI & $15.9(2.2)$ & $16.8(4.1)$ & $22.0(1.5)$ & $22.0(1.5)$ \\
\hline EDE-Q & $4.3(1.5)$ & $4.3(1.9)$ & $0.5(0.9)$ & $0.5(0.5)$ \\
\hline HADS_D & $9.5(6.3)$ & $9.5(5.8)$ & $0.0(1.5)$ & $0.0(3.0)$ \\
\hline HADS_A & $13.5(3.8)$ & $13.0(6.3)$ & $3.0(7.5)$ & $2.0(3.0)$ \\
\hline $\mathrm{OCl}-\mathrm{R}$ & 17.5 (11.3) & $17.0(12.0)$ & $5.0(7.0)$ & $2.0(5.0)$ \\
\hline
\end{tabular}

Embedded

figures test

Accuracy

SEF $\quad 100 \%(5.6) \quad 100 \%(5.6) \quad 100 \%(5.6) \quad 100 \%(5.6)$

CEF $\quad 66.7 \%(11.1) \quad 77.8 \%(5.6) \quad 72.2 \%(8.3) \quad 77.8 \%(19.4)$

Reaction time

\begin{tabular}{lllll} 
SEF & $1,743.8 \mathrm{~ms}$ & $1,628.3 \mathrm{~ms}$ & $1,566.6 \mathrm{~ms}$ & $1,337.3 \mathrm{~ms}$ \\
& $(1,137.9)$ & $(542.8)$ & $(423.2)$ & $(429.5)$ \\
CEF & $\begin{array}{llll}5,738.5 \mathrm{~ms} \\
(733.0)\end{array}$ & $\begin{array}{l}4,696.8 \mathrm{~ms} \\
(1,164.1)\end{array}$ & $\begin{array}{l}5,233.1 \\
(1,020.5)\end{array}$ & $\begin{array}{l}4,476.4 \mathrm{~ms} \\
\end{array}$ \\
\hline
\end{tabular}

Performance scores are given for both simple (SEF) and complex (CEF) embedded figures. Values given are in median (interquartile range). $E F T$ embedded figures test, $A N$ anorexia nervosa, $H C$ healthy controls, $T 1$ initial scan, $T 2$ follow-up, $B M I$ body mass index, EDE-Q Eating Disorder Examination Questionnaire, HADS Hospital Anxiety and Depression Scale, OCI-R Obsessive Compulsive Inventory Revised, SEF Simple Embedded Figures, CEF Complex Embedded Figures. 
Table 2 Cluster properties of brain regions showing a significant group by time interaction on CEF

\begin{tabular}{llllll}
\hline Region & Size (voxels) & \multicolumn{2}{l}{ Talairach coordinates } & Cluster $\boldsymbol{p}$ value (FDR corrected) \\
\hline & & $X$ & $Y$ & $Z$ & 0.003 \\
Left fusiform gyrus & 82 & -39.7 & -63.0 & -23.7 & 0.003 \\
Left middle occipital gyrus & 74 & -25.3 & -77.8 & -1.7 & 0.005 \\
Right medial frontal gyrus & 62 & 14.4 & -18.5 & 47.9 & \\
\hline
\end{tabular}

Coordinates are those of the cluster peak and are in the standard space of Talairach and Tournoux.

effect of group (SS $=336.11, \mathrm{df}=1, H=3.028, p=0.08)$ and time $(\mathrm{SS}=400.00, \mathrm{df}=1, H=3.604, p=0.06)$ on reaction time were found, while there were no differences on accuracy $(\mathrm{SS}=5.44, \mathrm{df}=1, H=0.049, p=0.82)$. On CEF, a main effect of time was found on reaction time $(S S=1,089.00$, $\mathrm{df}=1, H=9.818, p<0.001)$ as well as accuracy (SS $=608.44$, $\mathrm{df}=1, H=5.482, p=0.02$ ), but no main effect of group was seen ( $\mathrm{SS}=32.11, \mathrm{df}=1, H=1.025, p=0.31$ ).

Similar to the behavioural analysis, imaging data were analysed to assess group $\times$ time interactions on SEF and

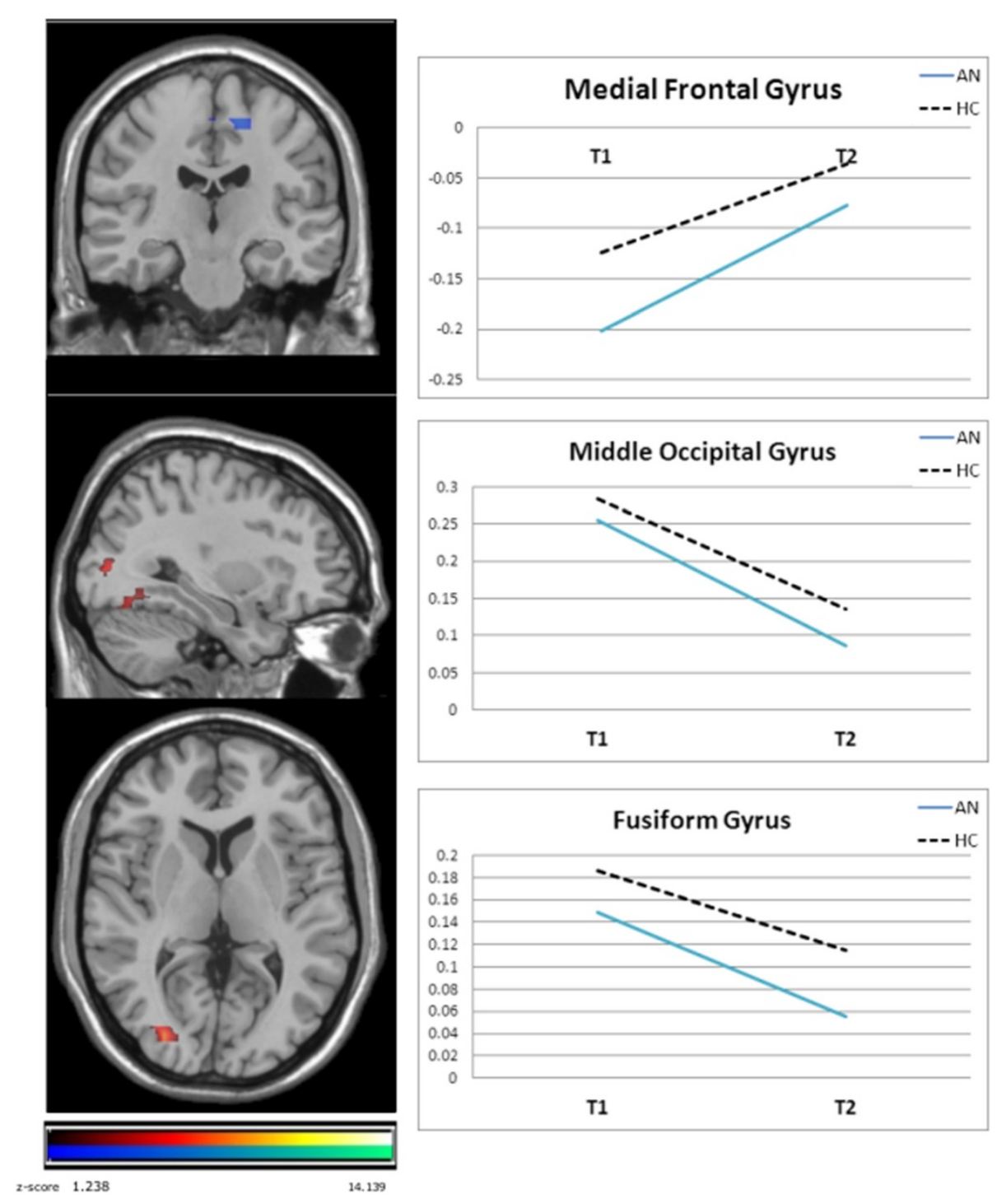

Figure 1 Changes in blood-oxygenation-level dependent response for complex embedded figures on the embedded figures test (EFT) illustrating a group $\times$ time interaction indicative of task-related activation (in red-yellow) and task-related deactivation (in blue-green) in those with anorexia nervosa (AN) and healthy controls (HC). A goodness of fit statistic (y-axis, arbitrary unit) was extracted from the cluster mean of reported brain regions on the EFT and plotted along the initial (T1) and follow-up (T2) scans ( $x$-axis) for both AN (in blue) and HC (in black, dotted). 
CEF. There was no indication of significant changes in blood-oxygenation-level dependent (BOLD) signal on SEF. On CEF, significant group $\times$ time interactions were found in the medial frontal gyrus (extending towards the precuneus), fusiform gyrus (extending into the cerebellum) and middle occipital gyrus (extending into the lingual gyrus (Table 2). In these regions, the average BOLD response was extracted (by averaging the underlying goodness of fit statistics) for further interpretation (Figure 1).

\section{Discussion}

The aim of this study was to assess the effects of CRT on central coherence in terms of performance and brain function. There was a clear improvement in performance on CEF with time, but this was not significantly different between the groups and likely to be the result of a practice effect. Neuroimaging data revealed a decrease in taskrelated activation in both groups and is in accordance with the notion of a practice effect. However, during CEF, there were stronger decreases in BOLD signal from $\mathrm{T} 1$ to $\mathrm{T} 2$ in $\mathrm{AN}$ compared to $\mathrm{HC}$ in the fusiform gyrus and middle occipital gyrus. Accordingly, AN demonstrated a greater decrease in task-related deactivation in the medial frontal gyrus at $\mathrm{T} 2$. The medial frontal lobes have been implicated in the default mode network [24], and previous studies have shown that task-related decreases negatively correlate with taskrelated activation. As such, the findings presented here are consistent with the notion of a shift in activation towards the cognitive demands of the task as task-related deactivation increases $[27,28]$. While the greater decrease in neural effort could be indicative of changes due to CRT, our design made ruling out differences in practice effect unattainable, and we recommend future studies to also compare CRT to treatment as usual.

In the variant of the EFT paradigm utilized here, the target shape is always present and participants have to make a choice between two figures. This is different from the original paradigm in which participants have to state whether or not the figure is present [25]. As such, the paradigm requires participants to switch their attention between two stimuli to search for the simple shape and utilize a more global strategy to successfully perform the task. Our previous cross-sectional fMRI findings on the EFT reported poorer performance in AN alongside greater task-related activation in the fusiform gyrus and greater task-related deactivation in the posterior cingulate cortex [19]. Their poorer performance was in line with previous literature on central coherence [6,7], and the changes in BOLD response suggested that those with AN do not direct their neural effort as efficiently as controls. This was interpreted as indicative of a cognitive bias towards detailoriented thinking and an inability to utilize a more global strategy. Many of the exercises in CRT focus on thinking in terms of the bigger picture instead of focusing on the details [21]. In light of this, the findings presented here could be indicative of a more efficient use of neural resources in those with AN after CRT.

This pilot study provides data for further research and has strengths and limitations which could be considered for future studies. To date, this is the first study to longitudinally assess central coherence using fMRI, before and after CRT, in those with AN and compare their performance to the general population. However, we are mindful of the limitations of this exploratory design, such as the absence of a parallel measure of the embedded figure test, a small sample size and a non-randomised design. Furthermore, our chosen statistical approach may have been too conservative for such a small sample size, and an increase of power in future studies might lead to clearer results. Though we were unable to ensure that antidepressant medication did not play a role in our findings, medication is part of a pragmatic and a realworld representation of $\mathrm{AN}$, and whether SSRIs have an effect in patients with low BMIs can be debated to some extent $[3,20]$.

In summary, the findings from larger CRT studies that have shown positive behavioural improvements have not been replicated in this study $[13,16,18]$. We do believe that while inconclusive, the conservative findings from this study validate the need to further extend research into brain functioning in AN. Future longitudinal studies using larger samples and comparing CRT to treatment as usual are required for a better understanding of the effects of CRT at a neural level.

\section{Competing interests}

The authors declare that they have no competing interests.

\section{Authors' contributions}

LF recruited some participants, carried out the analysis of the study and wrote the manuscript. VG provided advice on the analysis of data. NL participated in protocol writing and recruited most of the participants. HD recruited the participants and delivered the CRT. AS and SW participated in the design of the study and protocol meetings and helped draft the manuscript. KT obtained the funding, designed the study, recruited the participants, supervised the clinical and research work involved in this study, coordinated the study and helped draft the manuscript. All authors read and approved the final manuscript.

\section{Acknowledgements}

This work was supported by the NIHR Biomedical Research Centre for Mental Health at South London and Maudsley NHS Foundation Trust and Institute of Psychiatry, King's College London. KT would like to thank Swiss Anorexia Foundation and Maudsley Charity for the additional support for this study.

\section{Author details}

'Department of Psychological Medicine, King's College London, Institute of Psychiatry, De Crespigny Park, London SE5 8AF, UK. ²Department of Neuroimaging, King's College London, Institute of Psychiatry, London SE5 8AF, UK. ${ }^{3} \mathrm{NIHR}$ Biomedical Research Centre for Mental Health at South London and Maudsley NHS Foundation Trust Institute of Psychiatry, King's College London, London SE5 8AF, UK. ${ }^{4}$ Illia State University, Cholokashvili 5 , 0162 Tbilisi, Georgia. 
Received: 10 June 2014 Accepted: 1 September 2014

Published online: 16 September 2014

\section{References}

1. Hudson Jl, Hiripi E, Pope HG, Kessler RC: The prevalence and correlates of eating disorders in the National Comorbidity Survey Replication. Biol Psychiatry 2007, 61(3):348-358.

2. Arcelus J, Mitchell AJ, Wales J, Nielsen S: Mortality rates in patients with anorexia nervosa and other eating disorders. A meta-analysis of 36 studies. Arch Gen Psychiatry 2011, 68(7):724-731.

3. Steinhausen HC: Outcome of eating disorders. Child Adolesc Psychiatr Clin N Am 2009, 18(1):225-242.

4. Treasure J, Claudino AM, Zucker N: Eating disorders. Lancet 2010, 375(9714):583-593.

5. Tchanturia K, Lock J: Cognitive remediation therapy for eating disorders: development, refinement and future directions. Curr Top Behav Neurosci 2011, 6:269-287.

6. Lang K, Lopez C, Stahl D, Tchanturia K, Treasure J: Central coherence in eating disorders: an updated systematic review and meta-analysis. World J Biol Psychiatry 2014, 1:1-14.

7. Lopez C, Tchanturia K, Stahl D, Booth R, Holliday J, Treasure J: An examination of the concept of central coherence in women with anorexia nervosa. Int J Eat Disord 2008, 41(2):143-152.

8. Oldershaw A, Hambrook D, Stahl D, Tchanturia K, Treasure J, Schmidt U: The socio-emotional processing stream in anorexia nervosa. Neurosci Biobehav Rev 2011, 35(3):970-988.

9. Tchanturia K, Davies H, Roberts M, Harrison A, Nakazato M, Schmidt U, Treasure J, Morris R: Poor cognitive flexibility in eating disorders: examining the evidence using the Wisconsin Card Sorting Task. PLoS One 2012, 7(1):e28331.

10. Tchanturia K, Lloyd S, Lang K: Cognitive remediation therapy for anorexia nervosa: current evidence and future research directions. Int J Eat Disord 2013, 46(5):492-495.

11. Whitney J, Easter A, Tchanturia K: Service users' feedback on cognitive training in the treatment of anorexia nervosa: a qualitative study. Int J Eat Disord 2008, 41(6):542-550.

12. Lock J, Agras WS, Fitzpatrick KK, Bryson SW, Jo B, Tchanturia K: Is outpatient cognitive remediation therapy feasible to use in randomized clinical trials for anorexia nervosa? Int J Eat Disord 2013, 46(6):567-75.

13. Tchanturia K, Davies H, Lopez C, Schmidt U, Treasure J, Wykes T: Neuropsychological task performance before and after cognitive remediation in anorexia nervosa: a pilot case-series. Psychol Med 2008, 38(9):1371-1373.

14. Wexler BE, Anderson M, Fulbright RK, Gore JC: Preliminary evidence of improved verbal working memory performance and normalization of task-related frontal lobe activation in schizophrenia following cognitive exercises. Am J Psychiatry 2000, 157(10):1694-1697.

15. Wykes T, Brammer M, Mellers J, Bray P, Reeder C, Williams C, Corner J: Effects on the brain of a psychological treatment: cognitive remediation therapy: functional magnetic resonance imaging in schizophrenia. Br J Psychiatry 2002, 181:144-152.

16. Bor J, Brunelin J, d'Amato T, Costes N, Suaud-Chagny MF, Saoud M, Poulet E: How can cognitive remediation therapy modulate brain activations in schizophrenia? An fMRI study. Psychiatry Res 2011, 192(3):160-166.

17. Penades R, Pujol N, Catalan R, Massana G, Rametti G, Garcia-Rizo C, Bargalló $N$, Gastó $C$, Bernardo $M$, Junqué C: Brain effects of cognitive remediation therapy in schizophrenia: a structural and functional neuroimaging study. Biol Psychiatry 2013, 73(10):1015-1023.

18. Eack SM, Hogarty GE, Cho RY, Prasad KM, Greenwald DP, Hogarty SS, Keshavan MS: Neuroprotective effects of cognitive enhancement therapy against gray matter loss in early schizophrenia: results from a 2-year randomized controlled trial. Arch Gen Psychiatry 2010, 67(7):674-682.

19. Fonville L, Lao-Kaim NP, Giampietro V, Van den Eynde F, Davies H, Lounes N, Andrew C, Dalton J, Simmons A, Williams S, Baron-Cohen S, Tchanturia K: Evaluation of enhanced attention to local detail in anorexia nervosa using the embedded figures test; an FMRI study. PLoS One 2013, 8(5):e63964.

20. Tchanturia K, Davies H, Campbell IC: Cognitive remediation therapy for patients with anorexia nervosa: preliminary findings. Ann Gen Psychiatry 2007, 6:14.
21. Tchanturia $\mathrm{K}$, Davies $\mathrm{H}$, Reeder $\mathrm{C}$, Wykes $\mathrm{T}$ : Cognitive remediation for anorexia nervosa (manual). 2010 [http://www.national.slam.nhs.uk/wp-content/uploads/ 2014/04/Cognitive-remediation-therapy-for-Anorexia-Nervosa-Kate-Tchantura.pdf] [http://media.wix.com/ugd/2e1018_f71866481f9f44e5a342fb068b891a8c.pdf].

22. Ho DE, Imai K, King G, Stuart EA: Matching as nonparametric preprocessing for reducing model dependence in parametric causal inference. Polit Anal 2007, 15(3):199-236.

23. Ho D, Imai K, King G, Stuart E: Matchlt: nonparametric preprocessing for parametric casual inference. 2006. R package version, 2-2.

24. R Core Team: R: A Language and Environment for Statistical Computing. Vienna: R Foundation for Statistical Computing; 2013 [http://www.R-project.org].

25. Witkin H, Oltman PK, Raskin E, Karp SA: A Manual for the Embedded Figure Test. Menlo Park: Consulting Psychologists Press; 1971.

26. Corp IBM: IBM SPSS Statistics for Windows. Armonk: IBM Corp; 2011.

27. Scheirer CJ, Ray WS, Hare N: Analysis of ranked data derived from completely randomized factorial designs. Biometrics 1976, 32(2):429-434.

28. Sokal RR, Rohlf FJ: Biometry: The principles and Practice of Statistics in Biological Research. 3rd edition. New York: W.H. Freeman; 1995.

doi:10.1186/s12991-014-0025-z

Cite this article as: Fonville et al:: Cognitive remediation, brain function and central coherence: an anorexia nervosa pilot study. Annals of General Psychiatry 2014 13:25

\section{Submit your next manuscript to BioMed Central and take full advantage of:}

- Convenient online submission

- Thorough peer review

- No space constraints or color figure charges

- Immediate publication on acceptance

- Inclusion in PubMed, CAS, Scopus and Google Scholar

- Research which is freely available for redistribution

Submit your manuscript at www.biomedcentral.com/submit
C) Biomed Central 